\title{
Calidad del Currículo y la Deserción Universitaria en la Facultad de Ingeniería y Arquitectura de la Universidad Peruana Unión
}

\section{Quality of Curriculum and University Dropout in the Faculty of Engineering and Architecture of the Universidad Peruana Unión}

\author{
Vega Beteta, Enrique y Carbo Gil, Otto \\ Departamento de Matemática y Estadística. Facultad de Ingeniería y Arquitectura \\ - Universidad Peruana Unión \\ Recibido 10 de setiembre del 2015-Aceptado 26 de noviembre del 2015
}

\begin{abstract}
Resumen
El objetivo de la presente investigación fue determinar la relación existente entre la calidad de currículo y la deserción universitaria en la Facultad de ingeniería y Arquitectura de la UPeU, en el año 2013. El presente estudio corresponde al enfoque cuantitativo de tipo no experimental y de corte transversal. Enfoque cuantitativo, porque pretende medir las variables y presentar los resultados a través del tratamiento estadístico. Y es no experimental en tanto, no pretende manipular alguna variable, sino analizarlas en su contexto natural. De corte transversal, porque se aplicó los instrumentos en un momento único. Por el método de contrastación de hipótesis, corresponde al diseño correlacional múltiple, pues analiza las correlaciones que se dan entre los indicadores e ítems dentro de cada una de las dimensiones de cada variable. Es una investigación multivariada porque estudia la relación de más de dos variables tales como, Gestión administrativa, Calidad del currículo y la Deserción universitaria. Los datos fueron recogidos utilizando dos cuestionarios validaros, con un nivel de confiabilidad de alfa de cronbach de 0.89 y 0.82 . El cuestionario de Calidad de currículo y Deserción universitaria fue aplicado a 70 alumnos que se retiraron de la universidad. Los resultados muestran la relación existente entre la Calidad del currículo y la Deserción universitaria en sus diferentes dimensiones.
\end{abstract}

Palabras clave: Calidad del currículo, deserción universitaria.

\begin{abstract}
The aim of this research was to determine the relationship between the quality of curriculum and college dropout in the Faculty of Engineering and Architecture UPeU in 2013. This study is the quantitative approach of non-experimental and cross-sectional quantitative approach that aims to measure the variables and present the results through statistical treatment. And it's not as experimental, is not intended to manipulate some variable, but analyze them in their natural context. Cross section because the instruments was applied at a single time. By the method of hypothesis testing. It corresponds to multiple correlational design. Then it analyzes the correlations that exist between indicators and items within each of the dimensions of each variable. It is a multivariate research that studies the relationship of more than two variables such as administrative management, quality of curriculum and college dropout. Data were collected using two questionnaires validaros, with a level of reliability Cronbach's alpha of 0.89 and 0.82 . The questionnaire quality of curriculum and college dropout was applied to 70 students who withdrew from the university. The results show the relationship between the quality of curriculum and college dropout in different dimensions.
\end{abstract}

Keywords: Quality of curriculum, University dropout.

Correspondencia al autor:

email: kikevega@upeu.edu.pe,ottoc@upeu.edu.pe. 


\section{Introducción}

La presente investigación trata de determinar la relación existente entre la Calidad de currículo y la Deserción universitaria en la Facultad de Ingeniería y Arquitectura de la UPeU.

Comprender el término, calidad en la educación superior universitaria, requiere una explicación de las diferentes dimensiones y los ejes fundamentales, desde donde se puede reconocer la calidad de un sistema educativo.

La Universidad Peruana Unión forma parte del sistema educativo mundial de la Iglesia Adventista del Séptimo Día y como tal promueve el desarrollo integral del ser humano, en armonía con los principios y valores cristianos establecidos en las sagradas escrituras. Está dedicada a la formación especializada de profesionales creativos, investigadores y líderes que contribuyen al cumplimiento de la misión de la Iglesia y al desarrollo de la sociedad.

La Facultad de Ingeniería y Arquitectura forma parte de las cinco facultades que funciona en la Universidad Peruana Unión. La actividad formativa que ofrece la facultad en mención se logra a través de los programas académicos y de otras actividades académicas de carácter multidimensional que, en conjunto, constituyen un currículo integral al cual son expuestos los estudiantes universitarios.

Para ello, los programas y actividades académicas deben ser competitivos, interdisciplinarios, innovadores y prospectivos, donde se expresen el estado actual y las tendencias internacionales en ciencia, tecnología, cultura y práctica profesional.

Es evidente que el currículo es uno de los factores que más influyen en la calidad de la formación profesional universitaria, lo que conlleva al éxito de la institución.

En este contexto la calidad de la planificación, gestión, control y evaluación de los procesos curriculares, que implican aspectos externos (del contexto) e internos (institucionales), han de ser sincronizados en todos sus aspectos en términos de filosofía, políticas, objetivos, acciones y recursos institucionales y que requieren de condiciones de calidad en términos de cualidades y características que le confieren al currículo universitario, capacidad para satisfacer necesidades y expectativas implícitas y explícitas al sistema.
Otro factor que se analizo es las causas que originan la deserción universitaria, para lo cual lo distribuimos en las siguientes dimensiones: académicas, económicas, administrativas alas cuales consideramos como las más relevantes.

\section{Método}

El presente estudio corresponde al enfoque cuantitativo de tipo no experimental y de corte transversal. Enfoque cuantitativo, porque pretende medir las variables y presentar los resultados a través del tratamiento estadístico. $\mathrm{Y}$ es no experimental, en tanto no pretende manipular alguna variable, sino analizarlos en su contexto natural. De corte transversal, porque se aplicó los instrumentos en un momento único.

Por el método de contrastación de hipótesis, corresponde al diseño correlacional múltiple, pues analiza las correlaciones que se dan entre los indicadores e ítems dentro de cada una de las dimensiones de cada variable. Es una investigación multivariada, porque estudia la relación de dos variables tales como: Calidad del currículo y la Deserción universitaria.

El presente estudio se realizó en la Universidad Peruana Unión, para lo cual se seleccionó a la Facultad de Ingeniería y Arquitectura, de la Universidad Peruana Unión, con sus cinco Escuela Académicas:

a. Escuela Académica Profesional de Ingeniería de Sistema.

b. Escuela Académica Profesional de Ingeniería de Alimentos.

c. Escuela Académica Profesional de Ingeniería Ambiental.

d. Escuela Académica Profesional de Ingeniería Civil.

e. Escuela Académica Profesional de Arquitectura.

Incluyendo las filiales de Juliaca y Tarapoto donde funciona las escuelas académicas en mención. 
Vega, E. y Carbo, O.

Tabla 1

Muestra de estudio.

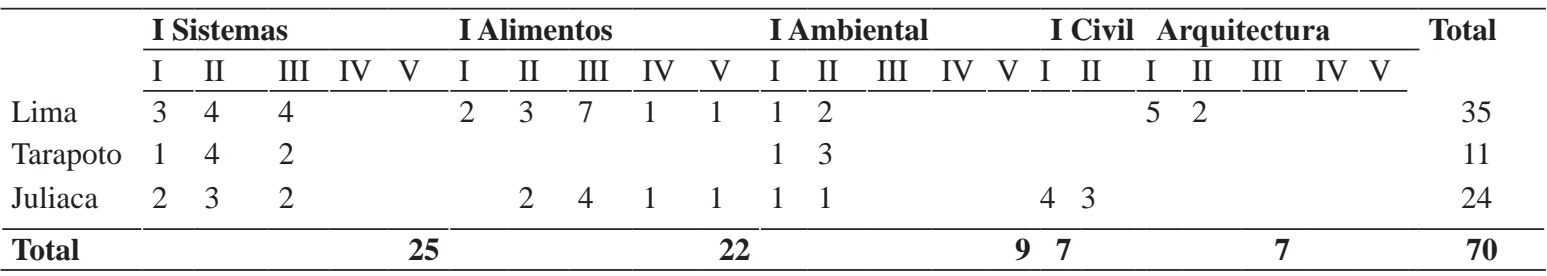

Para recopilar los datos de la presente investigación, se utilizó los siguientes instrumentos:

Para medir la variable 1: Calidad del currículo, se utilizará el instrumento para evaluar el currículo de una carrera profesional universitaria, elaborado por el Dr. Elías Mejía Mejia. Dicho instrumento fue adecuado y adaptado a una realidad diferente que consta de 62 preguntas, validada con un nivel de confiabilidad de alfa de Cronbach de 0.89 .

Para medir la Variable 2. Deserción de la población universitaria, se utilizará el cuestionario de determinantes de deserción universitaria, el cual consta de un cuestionario de 64 preguntas, validada con un nivel de confiabilidad de alfa de Cronbach de 0.82. Aplicada en las cinco escuelas académicas de la Facultad de Ingeniería y Arquitectura de la UPeU.

El procesamiento estadístico se desarrolló mediante el análisis del coeficiente de correlación, item por ítem.
Primero, se aplicó el instrumento a la muestra de investigación, procesando los datos en el programa spss 20.

Luego, se realizó un análisis exploratorio donde se descartó la relación de causalidad entre las variables y entre las dimensiones de las mismas. Lo que indujo a realizar el análisis ítem por ítem y entre indicadores, en cada una de las variables.

Posteriormente, se estableció las relaciones significativas entre los ítems, indicadores y dimensiones de cada variable.

En cuanto al análisis descriptivo, se presenta el comportamiento de los datos mediante tablas de frecuencia, ítem por ítem.

En cuanto al análisis inferencia, la prueba de hipótesis se realizó con el modelo estadístico Rho Sperman para variables ordinales.

\section{Resultados y Discusión}

Tabla 2

Correlación multivariada para muestras independientes entre la calidad de currículo y la deserción universitaria, política y estrategia administrativa, Área personal, Recursos económicos y logísticos, y el Liderazgo administrativo.

\begin{tabular}{lcc}
\hline KMO y Prueba de Bartlett & Estudiantes \\
\hline Medida de adecuación muestral de Kaiser-Meyer-Olkin. &, 835 \\
Prueba de esfericidad de Bartlett & Chi-cuadrado aproximado & 860,554 \\
& Gl & 6 \\
& Sig. &, 000 \\
\hline
\end{tabular}

Existe relación entre el plan de estudio, las sumillas, estructura del currículo, decisiones institucionales, objetivos, perfil profesional, área de desempeño laboral, marco filosófico y diagnóstico como dimensiones de la variable calidad de currículo.
No existe relación entre el plan de estudio, las sumillas, estructura del currículo, decisiones institucionales, objetivos, perfil profesional, área de desempeño laboral, marco filosófico y diagnóstico como dimensiones de la variable calidad de currículo. 
En cuanto a los estudiantes encuestados; los resultados obtenidos, en la tabla 2, permiten observar que el diagnóstico guarda relación directa y significativa con el marco filosófico, hallándose un coeficiente de correlación de 0.275 (28\%) y un p valor $=0.000<$ $0.05(\mathrm{p}<\alpha)$; lo cual significa que puede ser inferido en la población. Con el Perfil profesional en 0.248 $(25 \%)$ y un $\mathrm{p}$ valor $=0.000<0.05(\mathrm{p}<\alpha)$; con los objetivos en 0.202 (20\%) y un p valor $=0.000<0.05$ $(\mathrm{p}<\alpha)$; con las decisiones institucionales en 0.249 $(25 \%)$ y un $\mathrm{p}$ valor $=0.000<0.05(\mathrm{p}<\alpha)$; con la estructura curricular en $0.158(16 \%)$ y un p valor $=$ $0.002<0.05(\mathrm{p}<\alpha)$; con el plan de estudio en 0.157 $(16 \%)$ y un $p$ valor $=0.002<0.05(p<\alpha)$; lo cual significa que estos casos pueden ser inferidos en la población.

En cuanto al marco filosófico, se observa que guarda relación directa y significativa con el diagnóstico, hallándose un coeficiente de correlación de 0.275 (28\%) y un $\mathrm{p}$ valor $=0.000<0.05(\mathrm{p}<\alpha)$; con el Perfil profesional en 0.191 (19\%) y un p valor $=0.000<0.05$ $(\mathrm{p}<\alpha)$; con los objetivos en $0.268(27 \%)$ y un $\mathrm{p}$ valor $=0.000<0.05(\mathrm{p}<\alpha)$; con las decisiones institucionales en $0.270(27 \%)$ y un p valor $=0.000<0.05$ (p $<$ $\alpha)$; con la estructura curricular en $0.342(34 \%)$ y un $p$ valor $=0.000<0.05(\mathrm{p}<\alpha)$; con el plan de estudio en $0.242(24 \%)$ y un $p$ valor $=0.000<0.05(p<\alpha)$; con el sílabo en 0.275 (28\%) y un p valor $=0.000<0.05$ $(p<\alpha)$; lo cual significa que estos casos pueden ser inferidos en la población.

En cuanto al área docente, se observa que guarda relación indirecta y significativa con los objetivos, hallándose un coeficiente de correlación de $0.133(13 \%)$ y un $\mathrm{p}$ valor $=0.010<0.05(\mathrm{p}<\alpha)$; con la estructura curricular en $0.117(12 \%)$ y un p valor $=0.024<0.05$ $(p<\alpha)$; con el plan de estudio en $0.242(24 \%)$ y un $\mathrm{p}$ valor $=0.000<0.05(\mathrm{p}<\alpha)$; con el plan de estudio en $0.107(10 \%)$ y un $p$ valor $=0.038<0.05(p<\alpha)$; lo cual significa que estos casos pueden ser inferidos en la población.

En cuanto al perfil profesional, se observa que guarda relación directa y significativa con el diagnóstico, hallándose un coeficiente de correlación de 0.248 (25\%) y un $\mathrm{p}$ valor $=0.000<0.05(\mathrm{p}<\alpha)$; Con el marco docente en $0.191(19 \%)$ y un p valor $=0.000<0.05$ $(\mathrm{p}<\alpha)$; con los objetivos en $0.264(26 \%)$ y un $\mathrm{p}$ valor $=0.000<0.05(\mathrm{p}<\alpha)$; con las decisiones institucionales en $0.131(13 \%)$ y un p valor $=0.011<0.05(\mathrm{p}<$ $\alpha)$; con la estructura curricular en $0.278(28 \%)$ y un $p$ valor $=0.000<0.05(\mathrm{p}<\alpha)$; con el plan de estudio en
$0.172(17 \%)$ y un $p$ valor $=0.001<0.05(\mathrm{p}<\alpha)$; con el sílabo en $0.248(25 \%)$ y un p valor $=0.000<0.05$ $(\mathrm{p}<\alpha)$; lo cual significa que estos casos pueden ser inferidos en la población.

En cuanto a los objetivos, se observa que guarda relación directa y significativa con el diagnóstico, hallándose un coeficiente de correlación de $0.202(20 \%)$ $\mathrm{y}$ un $\mathrm{p}$ valor $=0.000<0.05(\mathrm{p}<\alpha)$; con el Marco filosófico en $0.268(27 \%)$ y un $p$ valor $=0.000<0.05$ $(\mathrm{p}<\alpha)$; área docente en $0.133(13 \%)$ y un $\mathrm{p}$ valor $=0.010<0.05(\mathrm{p}<\alpha)$; con el perfil profesional en $0.264(26 \%)$ y un $p$ valor $=0.000<0.05(p<\alpha)$; con los objetivos en 0.268 (27\%) y un p valor $=0.000$ $<0.05(\mathrm{p}<\alpha)$; con las decisiones institucionales en $0.178(18 \%)$ y un $p$ valor $=0.001<0.05(p<\alpha)$; con la estructura curricular en $0.306(30 \%)$ y un p valor $=$ $0.000<0.05(\mathrm{p}<\alpha)$; con el plan de estudio en 0.251 $(25 \%)$ y un $p$ valor $=0.000<0.05(p<\alpha)$; con el sílabo en $0.202(20 \%)$ y un $p$ valor $=0.000<0.05(p<\alpha)$; Lo cual significa que estos casos pueden ser inferidos en la población.

En cuanto a las decisiones institucionales, se observa que guarda relación indirecta y significativa con el diagnóstico, hallándose un coeficiente de correlación de $0.249(25 \%)$ y un p valor $=0.000<0.05(\mathrm{p}<\alpha)$; Con el Marco filosófico en 0.270 (27\%) y un p valor $=0.000<0.05(\mathrm{p}<\alpha)$; con el perfil profesional en $0.131(13 \%)$ y un $p$ valor $=0.011<0.05(p<\alpha)$; con los objetivos en $0.178(18 \%)$ y un p valor $=0.001$ $<0.05(\mathrm{p}<\alpha)$; con la estructura curricular en 0.259 $(26 \%)$ y un $\mathrm{p}$ valor $=0.000<0.05(\mathrm{p}<\alpha)$; con el plan de estudio en 0.385 (39\%) y un p valor $=0.000<0.05$ $(\mathrm{p}<\alpha)$; con el sílabo en $0.249(25 \%)$ y un $\mathrm{p}$ valor $=$ $0.000<0.05(\mathrm{p}<\alpha)$; lo cual significa que estos casos pueden ser inferidos en la población.

En cuanto a la estructura curricular, se observa que guarda relación directa y significativa con el diagnóstico, hallándose un coeficiente de correlación de $0.158(16 \%)$ y un $p$ valor $=0.002<0.05(p<\alpha)$; con el Marco filosófico en $0.242(342 \%)$ y un p valor $=$ $0.000<0.05(\mathrm{p}<\alpha)$; área docente en $0.117(12 \%)$ y un $\mathrm{p}$ valor $=0.024<0.05(\mathrm{p}<\alpha)$; con el perfil profesional en $0.278(28 \%)$ y un p valor $=0.000<0.05(p$ $<\alpha)$; con los objetivos en $0.306(30 \%)$ y un $\mathrm{p}$ valor $=$ $0.000<0.05(\mathrm{p}<\alpha)$; con las decisiones institucionales en $0.259(26 \%)$ y un p valor $=0.000<0.05$ (p $<$ $\alpha$ ); con el plan de estudio en $0.518(52 \%)$ y un $p$ valor $=0.000<0.05(\mathrm{p}<\alpha)$; con el sílabo en $0.158(16 \%)$ y un $\mathrm{p}$ valor $=0.002<0.05(\mathrm{p}<\alpha)$; lo cual significa que estos casos pueden ser inferidos en la población. 
Vega, E. y Carbo, O.

En cuanto al plan de estudio, se observa que guarda relación directa y significativa con el diagnóstico, hallándose un coeficiente de correlación de 0.157 (18\%) $\mathrm{y}$ un $\mathrm{p}$ valor $=0.002<0.05(\mathrm{p}<\alpha)$; con el Marco filosófico en $0.242(24 \%)$ y un $p$ valor $=0.000<0.05$ $(\mathrm{p}<\alpha)$; área docente en $0.107(10 \%)$ y un $\mathrm{p}$ valor $=0.038<0.05(\mathrm{p}<\alpha)$; con el perfil profesional en $0.172(17 \%)$ y un $\mathrm{p}$ valor $=0.001<0.05(\mathrm{p}<\alpha)$; con los objetivos en 0.251 (25\%) y un p valor $=0.000$ $<0.05(\mathrm{p}<\alpha)$; con las decisiones institucionales en $0.385(39 \%)$ y un $p$ valor $=0.000<0.05(p<\alpha)$; con la estructura curricular en 0.518 (52\%) y un $\mathrm{p}$ valor $=0.000<0.05(\mathrm{p}<\alpha) ;$ con el sílabo en $0.157(16 \%)$ y un $\mathrm{p}$ valor $=0.002<0.05(\mathrm{p}<\alpha)$; lo cual significa que estos casos pueden ser inferidos en la población. En cuanto a los sílabos, se observa que guarda relación indirecta y significativa con el Marco filosófico en $0.275(28 \%)$ y un p valor $=0.000<0.05(p<\alpha)$; con el perfil profesional en $0.248(25 \%)$ y un $p$ valor $=0.000<0.05(\mathrm{p}<\alpha)$; con los objetivos en 0.202 $(20 \%)$ y un $\mathrm{p}$ valor $=0.000<0.05(\mathrm{p}<\alpha)$; con las decisiones institucionales en $0.249(25 \%)$ y un p valor $=0.000<0.05(\mathrm{p}<\alpha)$; con la estructura curricular en $0.158(16 \%)$ y un $\mathrm{p}$ valor $=0.002<0.05(\mathrm{p}<\alpha)$; con el plan de estudio en $0.257(26 \%)$ y un p valor $=$ $0.002<0.05(\mathrm{p}<\alpha)$; lo cual significa que estos casos pueden ser inferidos en la población.

Tabla 3

Correlación de factores de la calidad de Currículo.

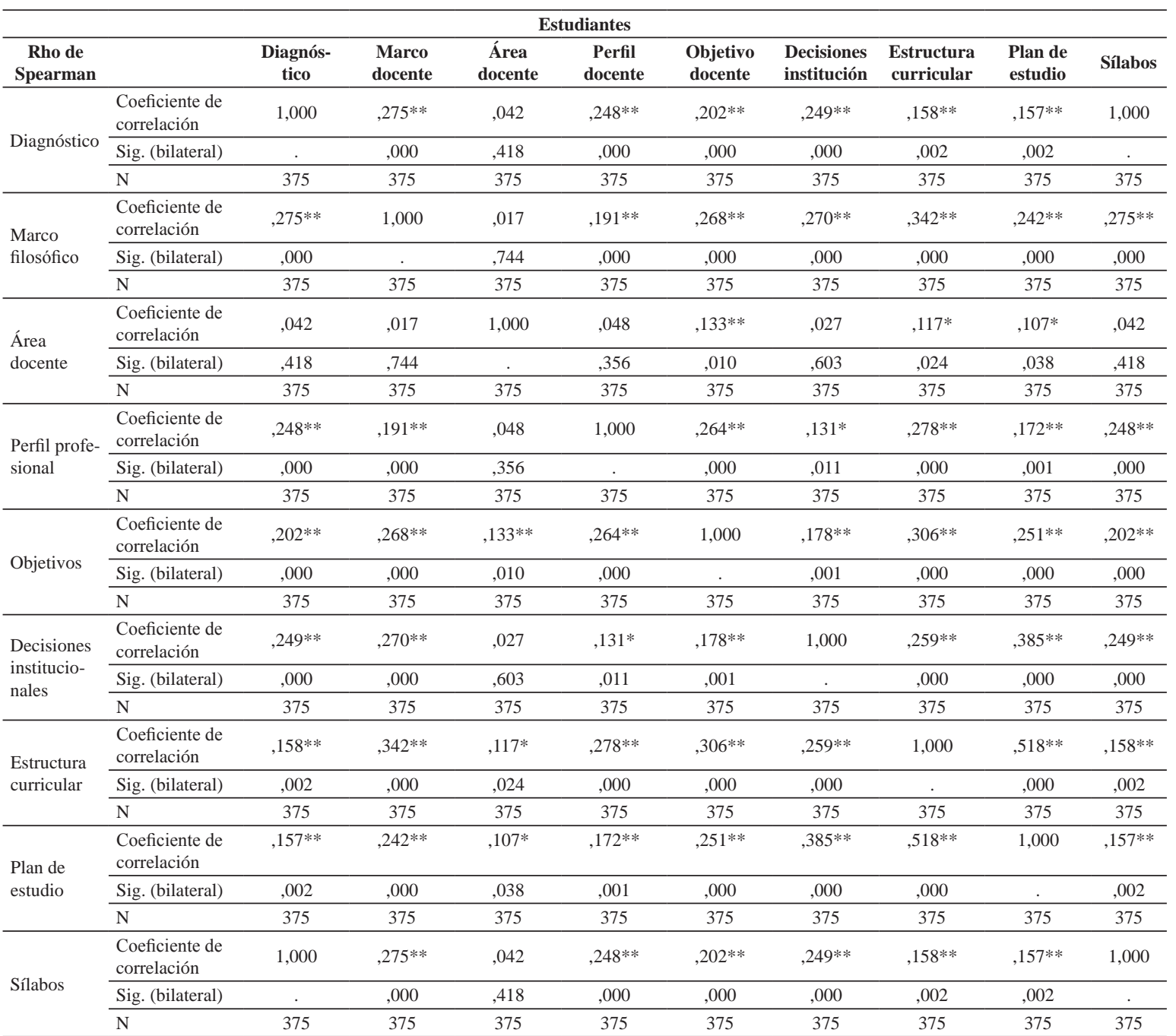




\section{Conclusiones}

En cuanto a los factores de la calidad del currículo, desde la perspectiva del estudiante, el diagnóstico guarda relación directa y significativa pero cuya relación es débil con el plan de estudio y los objetivos. Una correlación media con el plan de estudio y debajo del nivel medio con el marco filosófico, el perfil, profesional, las decisiones institucionales y la estructura curricular. En cuanto al marco filosófico, guarda relación directa y significativa, pero cuya relación es débil con el perfil profesional. Una correlación por debajo del nivel medio con el diagnóstico, con los objetivos, las decisiones institucionales, la estructura curricular, con el plan de estudio y con el sílabo. En cuanto al área docente, guarda relación directa y significativa pero cuya relación es débil con, los objetivos y con la estructura curricular. Una correlación por debajo del nivel medio con el plan de estudio. En cuanto al perfil profesional, guarda relación directa y significativa pero cuya relación es débil con el marco docente, las decisiones institucionales y el plan de estudio. Y una correlación por debajo del nivel medio con el diagnóstico, con los objetivos, la estructura curricular y con el sílabo. En cuanto a los objetivos, guarda relación directa y significativa pero cuya relación es débil con el diagnóstico, área docente, las decisiones institucionales y con el sílabo.

Y una correlación por debajo del nivel medio con el Marco filosófico, el perfil profesional, los objetivos, la estructura curricular y con el plan de estudio. En cuanto a las decisiones institucionales, guarda relación directa y significativa pero cuya relación es débil con los objetivos, y una correlación debajo del nivel medio con la estructura curricular, con el plan de estudio y con el sílabo. En cuanto a la estructura curricular, guarda relación directa y significativa pero cuya relación es débil con el diagnóstico, el Marco filosófico, el área docente y el sílabo. Una correlación media con el plan de estudio y debajo del nivel medio con el perfil profesional, los objetivos y las decisiones institucionales. En cuanto al plan de estudio, guarda relación directa y significativa pero cuya relación es débil con el diagnóstico, área docente, el perfil profesional y con el sílabo. Una correlación media con la estructura curricular y debajo del nivel medio con el marco filosófico, los objetivos y las decisiones institucionales. En cuanto a los sílabos, guarda relación directa y significativa pero cuya relación es débil con la estructura curricular y el plan de estudio. Y debajo del nivel medio con el Marco filosófico, el perfil profesional, las decisiones institucionales.
En cuanto a la prueba de KMO Kaiser-Meyer-Olkin, para correlaciones multivariadas de muestras independientes se observa que el plan de estudio, las sumillas, estructura del currículo, decisiones institucionales, objetivos, perfil profesional, área de desempeño laboral, marco filosófico y diagnostico como dimensiones de la variable calidad de currículo presentan un coeficiente de correlación considerable de 0.750. Asimismo, el p valor $=0.000<0.05(\mathrm{p}<\alpha)$ lo que significa que estos resultados pueden ser inferidos a la población de estudio.

En cuanto a los factores de la deserción universitaria, el factor académico no guarda relación significativa con el factor económico, pues los estudiantes que se retiran de la universidad por motivos económicos no lo hacen, a la vez, por motivos académicos. Asimismo, el factor académico y el factor de gestión administrativa no guardan relación significativa. Pues los estudiantes que se retiran de la universidad, por motivos económicos, no lo hacen a la vez por motivos administrativos. Luego, en cuanto a la relación entre el factor económico y el factor de gestión administrativa, se halló correlación indirecta y no significativa entre las variables, lo cual indica que los estudiantes que se retiran de la universidad por motivos económicos no lo hacen, a la vez, por motivos de gestión administrativa.

En cuanto a la prueba de KMO Kaiser-Meyer-Olkin, para correlaciones multivariadas de muestras independientes de deserción universitaria, se observa que el Aspecto Académico, Económico, Gestión administrativa presentan un coeficiente de correlación a nivel medio de 0.441 (44\%). Asimismo, el p valor $=0.152$ $>0.05(\mathrm{p}>\alpha)$ lo que significa que este resultado no puede ser inferido a la población de estudio.

Con la prueba de KMO Kaiser-Meyer-Olkin, para correlaciones multivariadas de muestras independientes, se observa que la calidad del currículo y la deserción universitaria presentan un coeficiente de correlación a nivel medio de 0.472 (47\%). Asimismo, el $\mathrm{p}$ valor $=0.326>0.05(\mathrm{p}>\alpha)$ lo que significa que estos resultados pueden ser inferidos a la población de estudio. 


\section{Referencias}

Agurto Calvo, S. (1998). Problemática universitaria. Ediciones de la Universidad Nacional Federico Villareal. Lima, Perú.

Álvarez, A. (1997). Compiladora, Hacia un currículo cultura. La vigencia de Vygotski en la Educación. Madrid, Gráficas Rogar.

Barbera, V. (1993). Cómo elaborar un proyecto curricular de centro sin dificultades añadidas. Madrid, Editorial Escuela Española.

Canales, A. \& Ríos, D. (2007). Factores explicativos de la deserción universitaria. Calidad en la Educación, 26, 171-201.

Castro Pereyra, M. (1986). La evaluación curricular, aproximación a un modelo. Lima.

Gimeno, J. (1991). El curriculum, una reflexión sobre la práctica. Morata. Madrid. España.

Kiernyezny, A. Calidad del currículo universitario. Universidad Nacional de Itapua.
Mirez, A. (2014). La deserción estudiantil en la Facultad de Ciencias Económicas y Empresariales de la Universidad de Piura, y su impacto en los resultados económicos. Tesis de pregrado en Administración de empresas. Universidad de Piura. Facultad de Ciencias Económicas y Empresariales. Programa Académico de Administración de empresas. Piura, Perú.

Peñaloza, W (1995). El currículo integral. Optimice editores. Lima.

Plascencia, R. (2011). Deserción universitaria preocupa al mundo.

Rojas, J. (2009). El abandono de los estudios: deserción y decepción de la juventud. Revista académica Hologramática, 10(4), 75-94. Recuperado de http://www.alfaguia.org/alfaguia/files/1319736384_13.pdf

Salcedo, A. (2011). Deserción universitaria en Colombia. Revista Academia y Virtualidad, 3(1), 50-60.

Sanabria, H. (2002). Deserción en estudiantes de enfermería en cuatro universidades del Perú. Anales de la Facultad de Medicina UNMAM. Lima. 63(4), 301-311. 